\title{
The Fine Structure of Bicomponent Polyester Fibers
}

\author{
S. HAIG ZERONIAN, ${ }^{1}$ MARIA K. INGLESBY, ${ }^{1}$ NING PAN, ${ }^{1}$ DAVID LIN, ${ }^{1}$ GENEVIEVE SUN, ${ }^{1}$ BHAVNA SONI, ${ }^{1}$ \\ KENNETH W. ALGER, ${ }^{1}$ JOHN D. GIBBON ${ }^{2, *}$ \\ ${ }^{1}$ Division of Textiles and Clothing, University of California, Davis, California 95616 \\ ${ }^{2}$ Core Fibers Research, Hoechst Celanese Corporation, Charlotte, North Carolina 28210
}

Received 11 March 1998; accepted 24 May 1998

\begin{abstract}
The application of alkaline hydrolysis to study the change in the fine structure of bicomponent polyester fibers as their surface is removed progressively was explored. The samples were prepared with a poly(butylene terephthalate) (PBT) sheath and a poly(ethylene terephthalate) (PET) core. The reagent used to hydrolyze the PBT was $1 M \mathrm{NaOH}$ in $75 / 25$ methanol to water since it appeared to react topochemically with the fiber. The solution reacted more rapidly with PET than with PBT. Thus, when necessary to retard the weight loss of the bicomponent fibers, after a 2 -h hydrolysis with this reagent to remove PBT, it was replaced with aqueous $1 M \mathrm{NaOH}$ solution containing $0.1 \%$ cetrimmonium bromide. Unlike homofil PET or PBT fibers, where alkaline attack appeared to be confined to the surface and left the residue relatively smooth, the bicomponent fiber was attacked unevenly, and penetration to the PET core occurred before all the PBT at the surface was removed. Nevertheless, most of the reaction was confined initially to the PBT sheath. The tenacity and extension at break of the PBT-PET fiber passed through a maximum as hydrolysis progressed. The fall in tenacity at high weight losses is ascribed to increasing surface defects in the fiber surface. After removal of the PBT by the hydrolysis, the birefringence of the residue became progressively higher. The synergistic effect of the PBT sheath on the properties of the PET core and the possible causes of the nonuniform hydrolysis at the PBT surface are discussed. An equation is proposed that includes an interaction parameter, which can be utilized to determine which property is affected most by the hydrolysis of a bicomponent fiber. In this instance, it appears from the parameters that the order is strength $>$ extension at break $\approx$ birefringence. () 1999 John Wiley \& Sons, Inc. J Appl Polym Sci 71: 1163-1173, 1999
\end{abstract}

Keywords: bicomponent fibers; poly(ethylene terephthalate); poly(butylene terephthalate); fine structure; morphology; physical properties; spinning

\section{INTRODUCTION}

We have shown that the fine structure of polyester fibers can be studied by hydrolyzing such products with aqueous sodium hydroxide. The re-

Correspondence to: S. H. Zeronian.

* Present address: 7000 Thermal Road, Charlotte, North Carolina 28211 (now retired).

Journal of Applied Polymer Science, Vol. 71, 1163-1173 (1999)

(C) 1999 John Wiley \& Sons, Inc.

CCC 0021-8995/99/071163-11 action starts at the fibers' peripheries, causing weight loss but leaving the residues nondegraded, as indicated by molecular weight determinations. ${ }^{1}$ The diameter of the residues can also be predicted from the weight losses. ${ }^{1}$ After the hydrolysis, such parameters as tenacity, extensibility, birefringence, and density can be determined, thus establishing a profile of the fine structure of the fiber. Using this technique, we have studied the properties of poly(ethylene terephthalate) (PET) fibers of intrinsic viscosities (IV) of about 


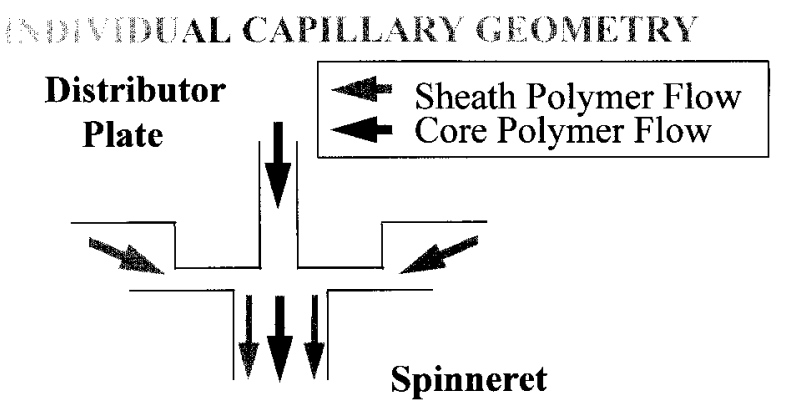

Figure 1 Schematic of the spinning equipment.

$0.6^{1,2}$ and $0.9^{3}$ as well as a thermotropic liquid crystalline copolyester fiber. ${ }^{4}$ Here, we explore application of this technique to sheath-core bicomponent fibers, in which the sheath is poly(butylene terephthalate) (PBT) and the core is PET, and to consider the effect on the physical properties of this material due to the presence of a PBT sheath on a PET core.

\section{EXPERIMENTAL}

\section{Materials}

The PBT-PET samples were spun on a machine specially designed for manufacturing sheathcore bicomponent fibers (Fig. 1). The PBT and PET polymers were melted in separate extruders and fed to a special spinning pack, which brought the two polymers together at the spinneret. Thus, the PBT polymer was kept cool until it met the PET stream immediately before exiting the pack as a filament. This minimized degradation of the $\mathrm{PBT}$ as the melt temperatures of the PET and PBT were 307 and $264^{\circ} \mathrm{C}$, respectively. The IVs of the starting PET and PBT were 0.92 and 1.00, respectively. Typically, the IV loss in spinning was about 0.04 . The sheath level was $25 \%$ by weight. PBT and PET homofil samples were produced on the same spinning machine, with no distinction being made between the sheath and core polymers. The PBT-PET samples were drawn in a two-stage operation to overall draw ratios of 2.6 and 2.2. The final draw roll was heated to $210^{\circ} \mathrm{C}$, and the yarn was allowed to relax $1.5 \%$ prior to winding up at $200 \mathrm{mpm}$. The spinning conditions and sample codes are given in Table I.

\section{Procedures}

\section{Alkaline Hydrolysis}

All hydrolyses were carried out in sealed flasks at $21^{\circ} \mathrm{C}\left( \pm 2^{\circ}\right)$ with mild mechanical agitation. The reaction solution varied depending on whether the yarn was composed of bicomponent filaments or homofils. The bicomponent samples were either hydrolyzed for progressively increasing lengths of time with $1 M \mathrm{NaOH}$ in 75/25 methanol to water or for $2 \mathrm{~h}$ with this reagent and subsequently for varying lengths of time with aqueous $1 M \mathrm{NaOH}$ containing $0.1 \%$ cetrimmonium bromide (CTAB). PBT homofil was hydrolyzed with $1.0 M \mathrm{NaOH}$ in $75 / 25$ methanol to water for varying lengths of time. In all cases, the reaction termination, sample rinsing, and drying were as described previously. ${ }^{1}$

\section{Tensile Testing}

Not less than 20 determinations per sample were made on single filaments on an Instron Testing Machine using a 101.6-mm gauge length at a crosshead speed of $20 \mathrm{~mm} / \mathrm{min}$ at $21^{\circ} \mathrm{C}$ and $65 \%$

Table I Samples and Codes

\begin{tabular}{lcccc}
\hline Sample Description & Code & $\begin{array}{c}\text { Spinning } \\
\text { Speed } \\
(\mathrm{m} / \mathrm{min})\end{array}$ & $\begin{array}{c}\text { Fverall } \\
\text { Draw Ratio }\end{array}$ & $\begin{array}{c}\text { Filament } \\
\text { Lin Density } \\
\text { (dtex) }\end{array}$ \\
\hline Undrawn PBT-PET & AU & 1600 & & 7.84 \\
Drawn PBT-PET & AD & 1600 & 2.6 & 3.29 \\
Undrawn PBT-PET & BU & 2000 & 2.2 & 7.18 \\
Drawn PBT-PET & BD & 2000 & & 3.06 \\
Undrawn PET & CU & 1600 & 2.6 & 7.14 \\
Drawn PET & CD & 1600 & 2.6 & 2.90 \\
Undrawn PBT & DU & 1600 & 2.59 \\
Drawn PBT & DD & 1600 & & 2.87 \\
\hline
\end{tabular}


relative humidity $(\mathrm{RH})$. The coefficient of variation of breaking forces, initial moduli, and extensions was about $10 \%$. Breaking forces were divided by the dtex of the filaments to convert the force to tenacity, where dtex is the weight in grams of 10,000 meters of filament conditioned at $21^{\circ} \mathrm{C}$ and $65 \% \mathrm{RH}$. For the starting products, it was measured by weighing $1-\mathrm{m}$ lengths of yarn and dividing by the number of filaments in the yarn. Ten determinations were made each time. The dtex of hydrolyzed products was calculated from that of the starting sample, and the weight loss suffered. Initial moduli were calculated from the slope of the beginning straight line portion of the force-extension curves.

\section{Birefringence}

Birefringence $(\Delta n)$ was measured by the Becke line method. Fibers were immersed in liquids of known refractive index (R. P. Cargille Laboratories, Inc.) and viewed under a Zeiss polarizing light microscope. Adjacent sections of the same fiber were used to obtain the refractive indices parallel to $\left(n_{\|}\right)$and perpendicular to $\left(n_{\perp}\right)$ the fiber axis, and $\Delta n$ was then calculated as follows:

$$
\Delta n=n_{\|}-n_{\perp}
$$

The birefringence of ten fibers was determined each time. Readings agreed to 0.001. With the starting drawn bicomponent fibers (AD and $\mathrm{BD}$ ), as well as $\mathrm{AD}$ and $\mathrm{BD}$ of low weight loss, a halo was observed at the fiber's edge, which made it difficult to make accurate determinations. Consequently, these birefringences are omitted from the tables.

\section{Sheath Thickness}

Drawn filament cross sections were cut by the Shirley plate method. ${ }^{5}$ The width of the sheath and fiber diameter were measured on a light microscope with a calibrated eyepiece. Determinations were made on ten filaments each time. The coefficient of variation of the diameters was about $3 \%$ and of the sheaths, $10 \%$.

\section{Density}

Measurements were made at $21^{\circ} \mathrm{C}\left( \pm 2^{\circ} \mathrm{C}\right)$ in a density gradient column prepared with aqueous calcium nitrate. ${ }^{6}$ Samples were in the form of bundles of fibers tied into small knots, and the loose ends were cut off. In the case of the PBT homofil samples, densities were determined by a technique essentially similar to that described for the calibration of standard floats in ASTM D1505-85. ${ }^{6}$

\section{Scanning Electron Microscopy}

Samples were examined with an International Scientific Instruments model DS 130 microscope operating in the secondary mode at an accelerating voltage of $10 \mathrm{kV}$. The samples were mounted on standard specimen stubs with silver paint, and the stubs were then spattered with gold.

\section{RESULTS AND DISCUSSION}

\section{Properties of Starting Products}

The diameters of the drawn bicomponent filaments $\mathrm{AD}$ and $\mathrm{BD}$ were $18.1 \mu \mathrm{m}$ and $17.6 \mu \mathrm{m}$, respectively, while the thicknesses of their sheaths were $1.61 \mu \mathrm{m}$ and $1.62 \mu \mathrm{m}$, respectively.

As expected, drawing of the samples increased their tenacity and initial modulus and reduced their extension at break (Table II). Birefringence is a measure of the orientation of the polymer chains with respect to the fiber axis. With respect to the undrawn bicomponent fibers, BU was spun at a faster speed than $\mathrm{AU}, 2000 \mathrm{~m} / \mathrm{min}$ versus $1600 \mathrm{~m} / \mathrm{min}$; and, as a result, the tenacity and birefringence of $\mathrm{BU}$ was higher than that of $\mathrm{AU}$.

Bicomponent AU and undrawn homofils CU and DU were spun at the same speed. The PBT homofil DU had a slightly lower tenacity than the PET homofil CU, but its birefringence was markedly higher, 0.109 versus 0.022 . In comparison, AU with its PBT sheath had a comparable tenacity to $\mathrm{CU}$, but its birefringence was significantly higher, 0.038 versus 0.022 . However, the birefringence of AU was much lower than that of DU. It appears then that the effect of the PET core of AU is to decrease the birefringence, or orientation, of its PBT sheath. It will be noted that birefringence determinations were being made at fiber peripheries.

After drawing, the tenacity and initial modulus of AD were similar to drawn PET homofil CD but $\mathrm{AD}$ had a lower extension at break than CD. Drawn PBT homofil DD had a markedly lower tenacity and initial modulus than either $\mathrm{AD}$ or $\mathrm{CD}$, while its extension at break was higher.

The lower initial modulus of DD compared to CD can be attributed, at least in part, to its 
Table II Tensile Properties, Birefringence, and Density of Starting Samples

\begin{tabular}{|c|c|c|c|c|c|}
\hline Code $^{a}$ & $\begin{array}{l}\text { Tenacity } \\
\text { (g/dtex) }\end{array}$ & $\begin{array}{c}\text { Extension } \\
\text { at Break } \\
(\%)\end{array}$ & $\begin{array}{l}\text { Initial } \\
\text { Modulus } \\
\text { (g/dtex) }\end{array}$ & Birefringence & $\begin{array}{r}\text { Density } \\
\left(\mathrm{g} / \mathrm{cm}^{3}\right)\end{array}$ \\
\hline $\mathrm{AU}$ & 2.26 & 170 & 19.8 & 0.038 & \\
\hline $\mathrm{AD}$ & 6.93 & 9.27 & 92.5 & b & 1.386 \\
\hline $\mathrm{BU}$ & 2.56 & 161 & 22.7 & 0.047 & \\
\hline $\mathrm{BD}$ & 7.48 & 9.21 & 103 & b & 1.387 \\
\hline $\mathrm{CU}$ & 2.27 & 190 & 19.4 & 0.022 & \\
\hline $\mathrm{CD}$ & 6.67 & 15.4 & 98.6 & 0.183 & 1.408 \\
\hline DU & 2.02 & 187 & 19.5 & 0.109 & 1.314 \\
\hline $\mathrm{DD}$ & 5.41 & 25.8 & 25.5 & 0.156 & 1.335 \\
\hline
\end{tabular}

${ }^{a}$ See Table I.

${ }^{\mathrm{b}}$ Not measured.

lower birefringence. Also, PBT has twice as many methylene groups per repeat unit than PET, and this also will affect modulus markedly. Ward et al. ${ }^{7}$ have associated the low modulus of PBT, as compared to PET, to the molecular conformation of PBT, never corresponding to the fully extended form so that deformation always involves bond angle rotations and bond bending rather than bond bending and stretching, as in the case of PET.

It appears that drawing does not increase the strength, initial modulus, and birefringence of DD, the homofil PBT sample, as much as the increases obtained with CD the homofil PET. In addition, it can be observed that the presence of a PET core permits the tenacity and initial modulus of bicomponent $\mathrm{AD}$ to be higher than that of DD and its extension at break to be lower.

\section{Hydrolysis Conditions}

From preliminary experiments, it appeared that the rate of hydrolysis of PBT products utilizing $2.5 \mathrm{M} \mathrm{NaOH}$ and $0.1 \% \mathrm{CTAB}$ at $21^{\circ} \mathrm{C}$ would be very slow. These are the conditions we have used in the past for other polyester products. ${ }^{1-4}$ Consequently, we initiated use of $1.0 M \mathrm{NaOH}$ in $75 / 25$ methanol to water at $21^{\circ} \mathrm{C}$ for the hydrolysis of PBT samples. When the drawn PBT homofil was hydrolyzed with this reagent, it was found that the weight loss increased linearly with time (Fig. 2). The $R^{2}$ value of the regression line was 0.990 . Also, the tenacity of the hydrolyzed residues only decreased a small amount up to a weight loss of 37\% (Table III). Thus, it appears, as in the case of the hydrolysis of PET with $2.5 \mathrm{M}$ $\mathrm{NaOH}$ and $0.1 \% \mathrm{CTAB}^{1-3}$ that the PBT is being hydrolyzed at the periphery of the fiber and the residue has been little affected.

In the case of the bicomponent filaments, once some of the PBT sheath had been eliminated, the residue was rapidly consumed by the sodium hydroxide dissolved in aqueous methanol. Consequently, in some instances, after a 2-h treatment with $1.0 M \mathrm{NaOH}$ in methanol to water had resulted in about $14 \%$ weight reduction, the reagent was replaced with aqueous $1 M \mathrm{NaOH}$ and $0.1 \%$ CTAB. The weight loss then proceeded more slowly and, thus, could be better controlled. For similar weight losses, the physical properties of the bicomponent fibers did not appear to be affected by the method of hydrolysis.

As stated earlier, the sheath level was $25 \%$ by weight. The question of why the hydrolysis rate increased, after $14 \%$ weight loss, on continued treatment with the $\mathrm{NaOH}-\mathrm{CH}_{3} \mathrm{OH}-\mathrm{H}_{2} \mathrm{O}$ reagent is interesting. It has been reported that PBT and PET have good miscibility and can form a common amorphous mixed phase. ${ }^{8,9}$ However, it is unlikely in our case that there was much blending at the interface since the contact time of the PBT and PET in the melt phase was only 1 to $2 \mathrm{~s}$. Studying cross sections by light microscopy, it could be observed that the demarcation between the sheath and core was sharp. Thus, a common amorphous mixed phase would not explain the increasing hydrolysis rate observed.

To attempt to obtain an explanation for the unanticipated acceleration of the hydrolysis after low weight losses, PBT-PET samples were examined using a scanning electron microscope (SEM). The surfaces of the nonhydrolyzed $\mathrm{AD}$ and $\mathrm{BD}$ were smooth, as shown in a typical micrograph 


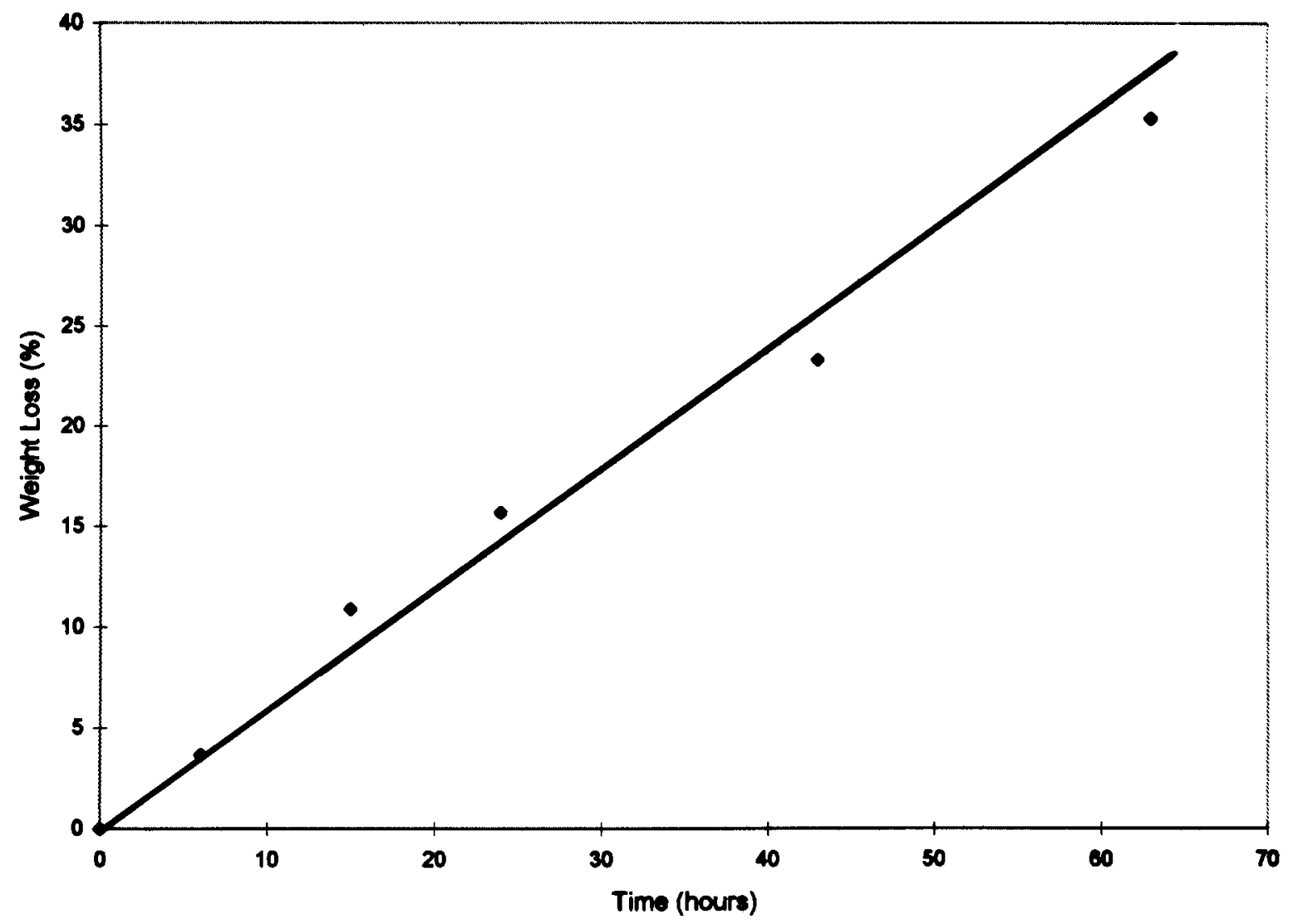

Figure 2 The weight loss-time relation of PBT homofil on hydrolysis with $1 M \mathrm{NaOH}$ in $75 / 25$ methanol to water.

(Fig. 3). On hydrolysis, neither $\mathrm{AD}$ or $\mathrm{BD}$ eroded uniformly, as expected, at the fiber surface. It will be observed that the reaction is occurring preferentially in some regions, leaving the appearance of the fiber as a trunk threading a set of rings, which presumably consists of PBT (Figs. 4 and 5). This contrasts markedly with the hydrolysis of the drawn PBT homofil (DD) (Figs. 6 and 7), which has a roughened surface similar to that of PET hydrolyzed with methanolic sodium hydroxide. ${ }^{10}$ As in the case of the nonhydrolyzed bicomponent fibers, DD had a smooth surface before hydrolysis (Fig. 8). It appears then that some regions of the PBT-PET bicomponent fibers are

Table III Weight Loss and Tenacity of PBT Homofil (DD) Hydrolyzed with 1.0M NaOH in 75/25 Methanol to Water at $21^{\circ} \mathrm{C}$ for Increasing Lengths of Time

\begin{tabular}{ccccc}
\hline $\begin{array}{c}\text { Hydrolysis } \\
\text { Time } \\
(\mathrm{h})\end{array}$ & $\begin{array}{c}\text { Weight Loss } \\
(\%)\end{array}$ & dtex & $\begin{array}{c}\text { Tenacity } \\
(\mathrm{g} / \text { dtex })\end{array}$ & $\begin{array}{r}\text { Relative } \\
\text { Tenacity }\end{array}$ \\
\hline- & - & 2.87 & 5.41 & 1.00 \\
- & 3.68 & 2.76 & 5.33 & 0.99 \\
15 & 10.9 & 2.56 & 5.26 & 0.97 \\
24 & 15.7 & 2.42 & 5.05 & 0.93 \\
43 & 23.3 & 2.20 & 5.04 & 0.93 \\
63 & 35.3 & 1.86 & 5.06 & 0.94 \\
80 & 36.6 & 1.82 & 4.95 & 0.91 \\
\hline
\end{tabular}




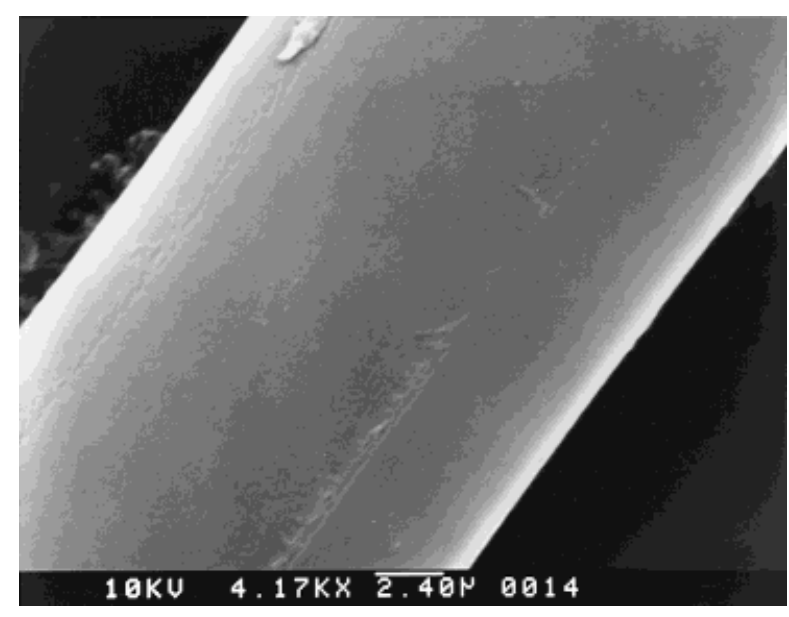

Figure 3 Scanning electron micrograph of nonhydrolyzed BD.

more reactive to the $\mathrm{NaOH}-\mathrm{CH}_{3} \mathrm{OH}-\mathrm{H}_{2} \mathrm{O}$ reagent than other sections, and penetration to the PET occurs at roughly $14 \%$ weight loss, resulting in an accelerating reaction as the PET is attacked in these areas.

At a weight loss of $3.8 \%$, some of the fiber's surfaces appeared porous (Fig. 9). Other sections already had the initiation of PBT rings (Fig. 10). At a weight loss of $36.8 \%$, the rings had disappeared, but there still appeared to be PBT on some of the fibers (Figs. 11 and 12), although the density of these products is now similar to that of a drawn PET (compare the densities of hydrolyzed $\mathrm{AD}$ in Table IV and the density of CD in Table II). At this point, preferential attack of the PET is taking place, leaving a skin of PBT. The

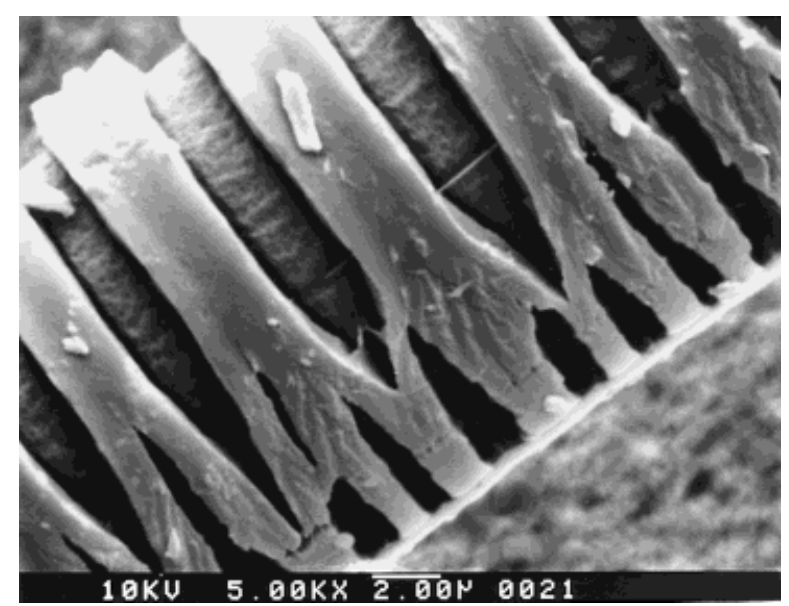

Figure 4 Scanning electron micrograph of hydrolyzed $\mathrm{BD}$; weight loss $=14.6 \%$.

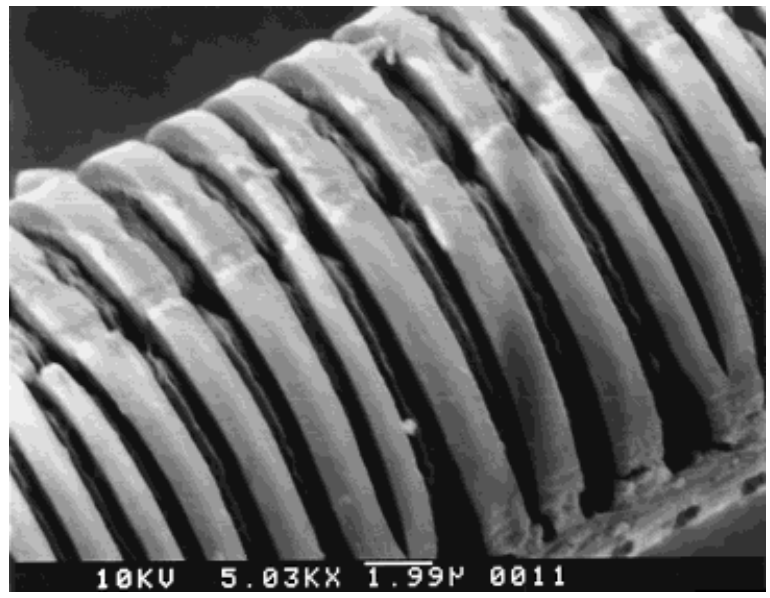

Figure 5 Scanning electron micrograph of hydrolyzed $\mathrm{AD}$; weight loss $=14.4 \%$.

surface of the fibers, when presumably only PET remains, can be observed in Figure 13, taken at a weight loss of $82.7 \%$.

The ring structure observed in Figures 4 and 5 reflect the morphology produced in the formation of the fiber. The following speculation is offered as an explanation. In the bicomponent spinning process, the PET melt temperature was kept about $10^{\circ}$ lower than normal. In normal spinning, this could give rise to melt fracture, namely, a slip stick phenomenon at the spinneret hole, which manifests itself in mild form as a sharkskin effect on the fiber surface. It also makes it difficult to take up the fibers. In the case of the bicomponent, the "slip stick" is covered by the PBT sheath and allows the fiber to apparently spin normally. In this regard, it was observed that the bicomponent

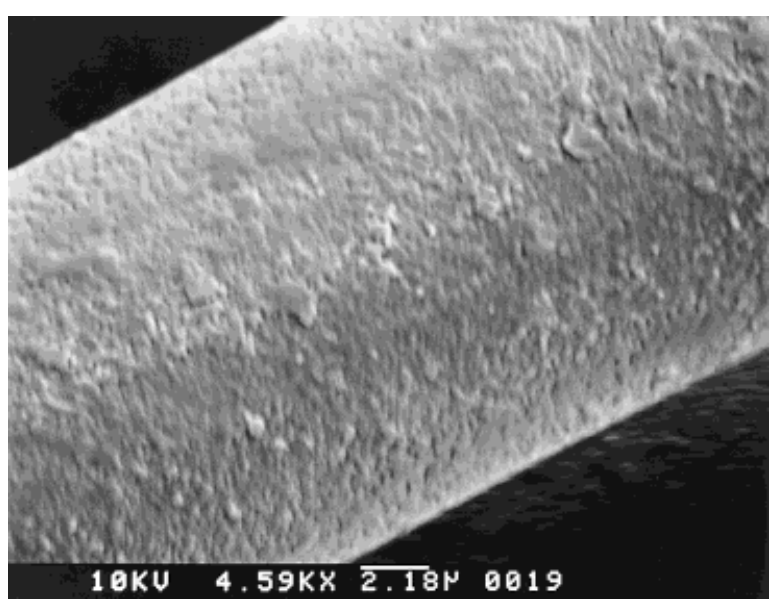

Figure 6 Scanning electron micrograph of hydrolyzed DD; weight loss $=15.7 \%$. 


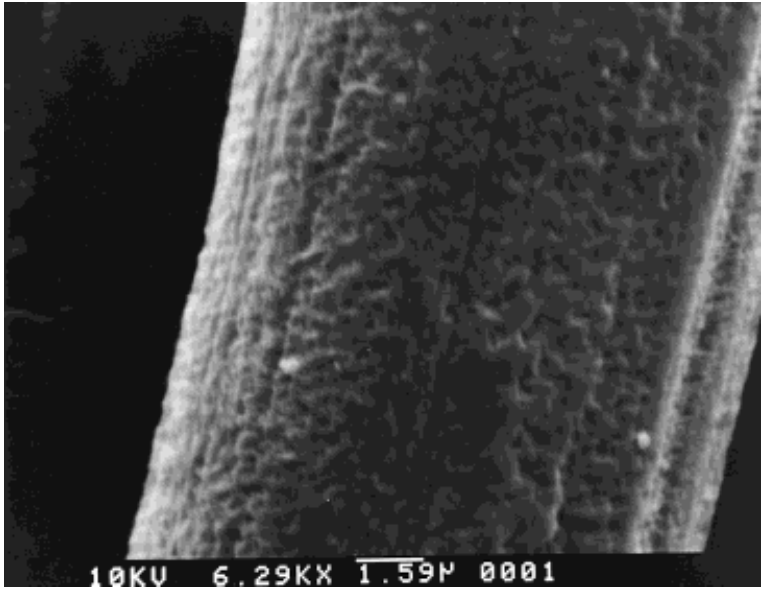

Figure 7 Scanning electron micrograph of hydrolyzed DD; weight loss $=38.5 \%$.

appeared to spin better than the PET alone. There were fewer filament breaks, as measured over long spinning periods. Thus, the melt fracture could create a crenelated PET core. As a consequence, the PBT sheath would have thick and thin regions. On hydrolysis with the methanolic $\mathrm{NaOH}$ solution, the PET core would be reached faster in areas where the PBT was thinner, and the resulting product would have the ringed morphology, we found.

Prior to the fiber formation, it was speculated that spinning at a lower than normal temperature for PET would significantly enhance the fiber properties of the bicomponent fiber compared to the homofil. While this did not happen, the bicomponent fiber was still comparable in tenacity with the PET (cf AD and CD, Table II). This indicates

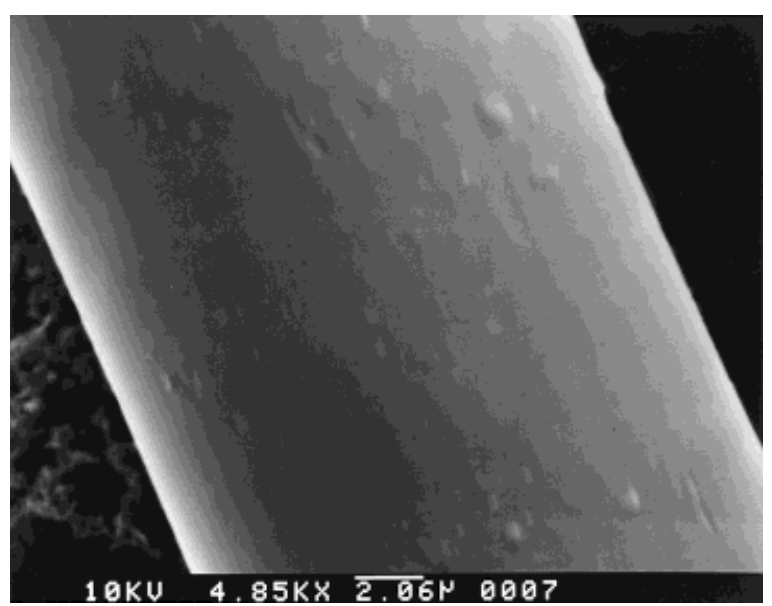

Figure 8 Scanning electron micrograph of nonhydrolyzed DD.

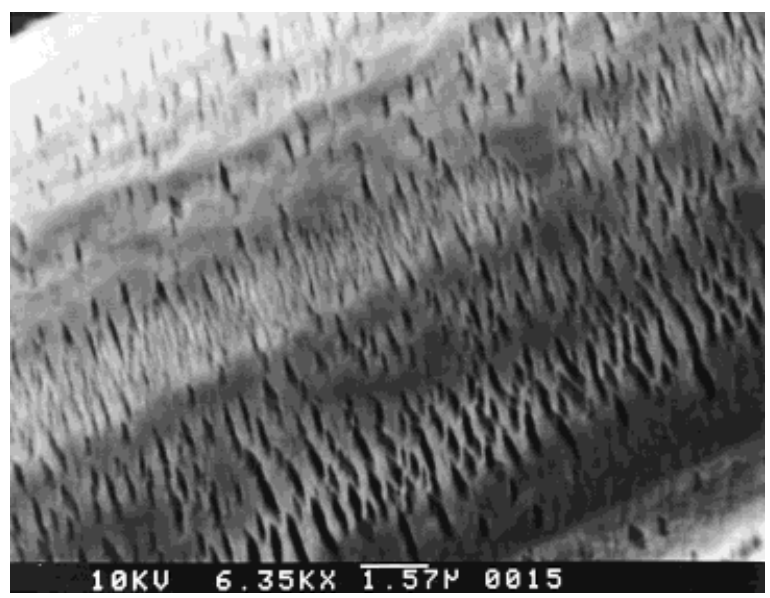

Figure 9 Scanning electron micrograph of hydrolyzed $\mathrm{AD}$; weight loss $=3.8 \%$.

that even though the fiber might be defective in that the PBT-PET interface is nonuniform, it is still strong. If conditions could be found to decrease this nonuniformity, then the fiber strength could be improved. The hydrolysis procedure described in this article could be used to measure the degree of improvement made by process changes.

\section{Tensile Properties and Birefringence of Drawn Bicomponent Fibers}

As stated earlier, the birefringence of $\mathrm{AD}$ and $\mathrm{BD}$, as well as $\mathrm{AD}$ and $\mathrm{BD}$ of low weight losses, could not be measured accurately. It will be observed that the birefringence of $\mathrm{AD}$ and $\mathrm{BD}$ appears to increase as the weight loss is pro-

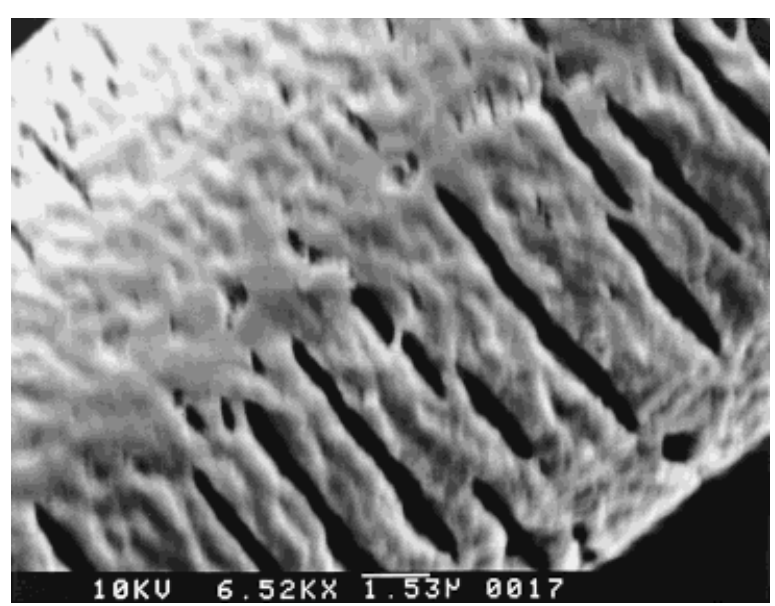

Figure 10 Scanning electron micrograph of hydrolyzed $\mathrm{AD}$; weight loss $=3.8 \%$. 


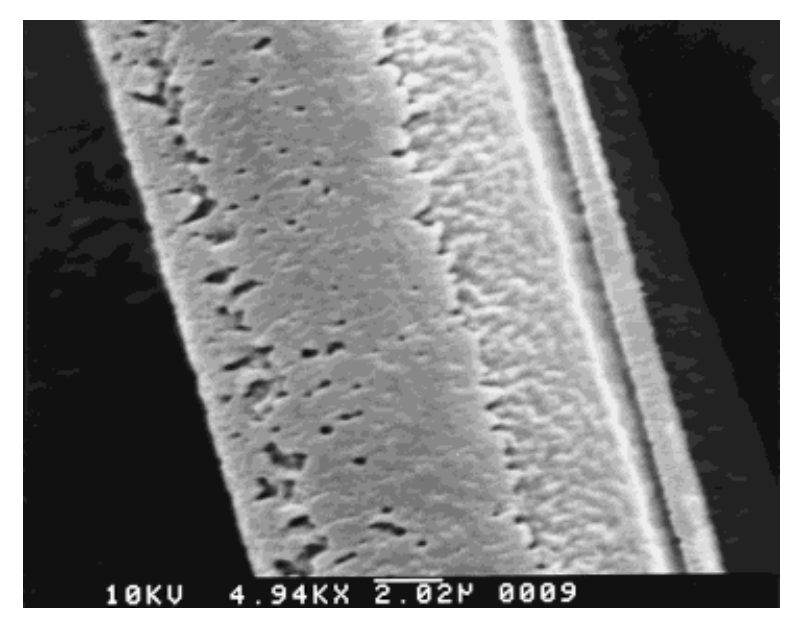

Figure 11 Scanning electron micrograph of hydrolyzed $\mathrm{AD}$; weight loss $=36.8 \%$.

gressively increased from 11 to $83 \%$. At the highest weight losses, the birefringence is similar to that of the drawn PET homofil CD (compare Tables II and IV).

The tenacity of both $\mathrm{AD}$ and $\mathrm{BD}$ passed through a maximum with increasing weight loss (Table V). This contrasts with the tenacity of homofil PET, which does not increase on loss in weight induced by alkaline hydrolysis. Instead, the tenacity of homofil PET will either remain constant or decrease slightly until the weight loss becomes large. ${ }^{1-3}$ Thus, removal of the PBT sheath results in a high tenacity PET core fiber. The sheath does not appear to have affected the breaking extension as markedly. Again, comparisons can be made between $\mathrm{AD}$

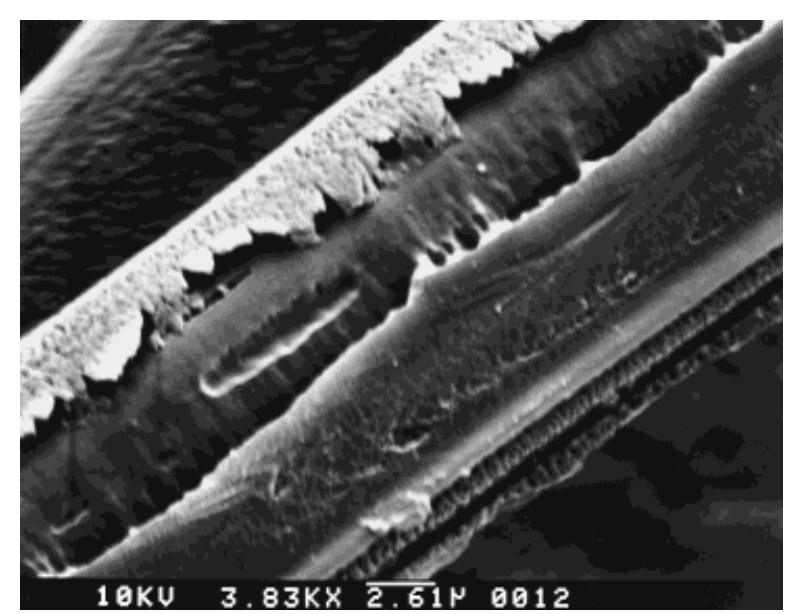

Figure 12 Scanning electron micrograph of hydrolyzed $\mathrm{AD}$; weight loss $=36.8 \%$.

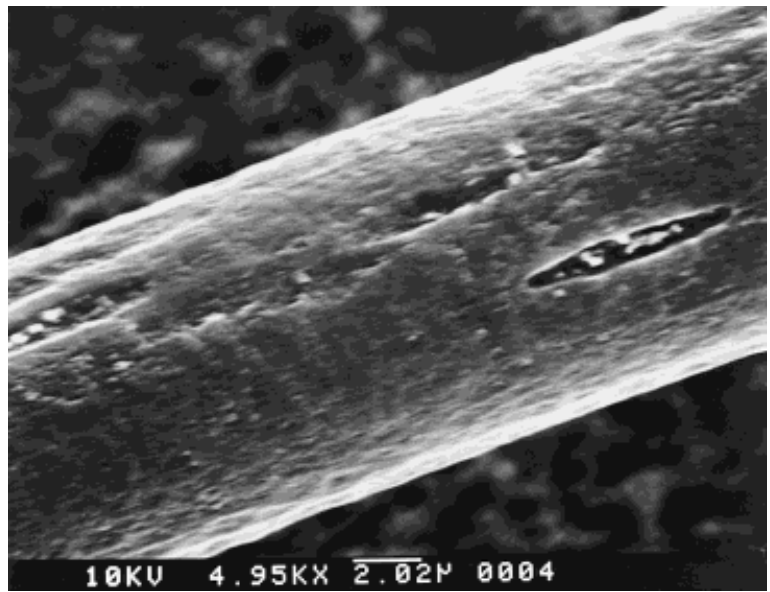

Figure 13 Scanning electron micrograph of hydrolyzed $\mathrm{AD}$; weight loss $=82.7 \%$.

and CD. Here, it will be noted the starting AD and $\mathrm{CD}$ have similar tenacities. By a weight loss of $30 \%, \mathrm{AD}$ had a markedly higher tenacity than CD (8.5 versus $6.7 \mathrm{~g} / \mathrm{tex}$ ). The fall in strength at high weight losses of $\mathrm{AD}$ may be due to increasing numbers of surface defects at which rupture is initiated. In previous work, ${ }^{1}$ it was shown that the DP of PET fiber subjected to aqueous alkaline hydrolysis remained constant, but its strength began to fall sharply after the weight loss increased above roughly $30 \%$. It was hypothesized that the fall in strength was due to increased weak points or defects at the surface. It is important to note, however, that in the present case when the PBT sheath appears to have a synergistic effect on the PET core, the loss in strength of the $\mathrm{AD}$ core is relatively small compared to CD, with the PET homofil spun under identical conditions. It is $13 \%$ at a weight loss of $82.7 \%$. In contrast, in the earlier study ${ }^{1}$ with a semidull and bright PET, the loss in strength of the former at a weight loss of $68 \%$ was $42 \%$; and in the latter case, the strength loss was $38 \%$ at $80 \%$ weight loss.

The initial modulus of $\mathrm{AD}$ also increased on hydrolysis at higher weight losses and became similar to that of CD (compare Tables II and V). Thus, the presence of the PBT sheath appeared to lower the overall initial modulus of the fiber; after its removal, the modulus of the residue reverted to that of PET. It appears from the data that the initial presence of a PBT sheath had resulted in a PET core with enhanced strength.

While attention has been focused on $\mathrm{AD}$ since it was spun under the same conditions as CD and 
Table IV Birefringence $(\Delta n)$ and Density of Drawn Bicomponent Filaments After Hydrolysis

\begin{tabular}{cccccc}
\hline \multirow{2}{*}{$\begin{array}{c}\text { Weight Loss } \\
(\%)\end{array}$} & $n_{\|}$ & $n_{\perp}$ & $\Delta n$ & $\begin{array}{c}\text { Density } \\
\left(\mathrm{g} / \mathrm{cm}^{3}\right)\end{array}$ \\
\hline \multirow{2}{*}{$\mathrm{AD}$} & - & & & $\mathrm{b}$ & 1.386 \\
& 0.60 & & & $\mathrm{~b}$ & \\
& 3.8 & & & $\mathrm{~b}$ & \\
& 11.2 & 1.723 & 1.544 & 0.179 & \\
& 14.4 & 1.723 & 1.544 & 0.179 & \\
& 29.6 & 1.725 & 1.540 & 0.185 & 1.400 \\
& 31.7 & 1.717 & 1.540 & 0.177 & 1.400 \\
$\mathrm{BD}$ & 36.8 & 1.728 & 1.540 & 0.188 & \\
& 82.7 & 1.724 & 1.541 & 0.183 & \\
& - & & & $\mathrm{b}$ & \\
& 0.77 & & & $\mathrm{~b}$ & \\
& 14.3 & 1.725 & 1.542 & 0.183 & \\
& 14.6 & 1.730 & 1.542 & 0.188 & \\
& 28.8 & 1.725 & 1.540 & 0.185 & \\
& 32.9 & 1.726 & 1.540 & 0.186 & \\
\hline
\end{tabular}

${ }^{\text {a }}$ Code given in Table I.

${ }^{\mathrm{b}}$ Not measured.

$\mathrm{DD}$, it will be noted that the change in properties of $\mathrm{BD}$ is similar to $\mathrm{AD}$ on hydrolysis (Tables IV and $\mathrm{V}$ ).

Table V Tensile Properties of Drawn Bicomponent Filaments After Hydrolysis

\begin{tabular}{ccccc}
\hline \multirow{2}{*}{ Sample $^{\mathrm{a}}$} & $\begin{array}{c}\text { Weight } \\
\text { Loss } \\
(\%)\end{array}$ & $\begin{array}{c}\text { Tenacity } \\
(\mathrm{g} / \mathrm{dtex})\end{array}$ & $\begin{array}{c}\text { Extension } \\
\text { at Break } \\
(\%)\end{array}$ & $\begin{array}{c}\text { Initial } \\
\text { Modulus } \\
(\mathrm{g} / \mathrm{dtex})\end{array}$ \\
\hline \multirow{2}{*}{$\mathrm{AD}$} & - & 6.93 & 9.27 & 92.5 \\
& 0.6 & 7.06 & 9.37 & 83.3 \\
& 3.8 & 7.18 & 9.16 & 81.3 \\
& 11.2 & 6.54 & 9.28 & 75.8 \\
& 14.4 & 7.01 & 9.99 & 94.6 \\
& 22.6 & 7.19 & 8.26 & 87.2 \\
& 25.0 & 7.69 & 9.33 & 98.3 \\
& 29.6 & 8.51 & 10.33 & 97.4 \\
& 31.7 & 7.90 & 9.80 & 104 \\
& 82.7 & 5.78 & 6.66 & 112 \\
& - & 7.48 & 9.21 & 103 \\
& 14.6 & 7.85 & 9.85 & - \\
& 22.1 & 8.11 & 8.26 & 92.2 \\
& 28.8 & 8.76 & 9.35 & - \\
& 30.5 & 8.60 & 8.58 & 104 \\
& 31.4 & 7.72 & 8.87 & 108 \\
& 32.9 & 9.22 & 9.23 & - \\
& 71.1 & 6.59 & 7.28 & 106 \\
\hline
\end{tabular}

${ }^{\text {a }}$ Code given in Table I.

\section{Analysis of the Properties as a Blended System}

Many properties of a material system blended with two or more different components can be calculated directly by using the simple Rule of Mixtures (ROM) when there is no interaction existed between the components. ${ }^{11}$ So, for a twoconstituent system, the overall system property $X_{s}$ can be found as

$$
X_{s}=X_{1} V_{1}+X_{2} V_{2}
$$

where $X_{i}$ and $V_{i}$ are the corresponding property, and the volume fraction of component $i=1$ and 2 .

From this relationship, it can be seen that if there is no interaction, the overall system property $X_{s}$ cannot be either greater or smaller than the property value $X_{i}$ of either component. Therefore, equation (1) can be used as a criterion to examine any possible interactions between the two constituents in a blended system. A plot of $X_{s}$ against $V_{1}$ results in a straight line. Thus, if the experimental results of the system property fall exactly on the line, it can be inferred that there are no interactions at all. If interactions indeed exist and cause a reduction in system property, then the data will fall below the line. On the other hand, an increase (a synergy) in system property will result in the experimental data occurring above the line. 


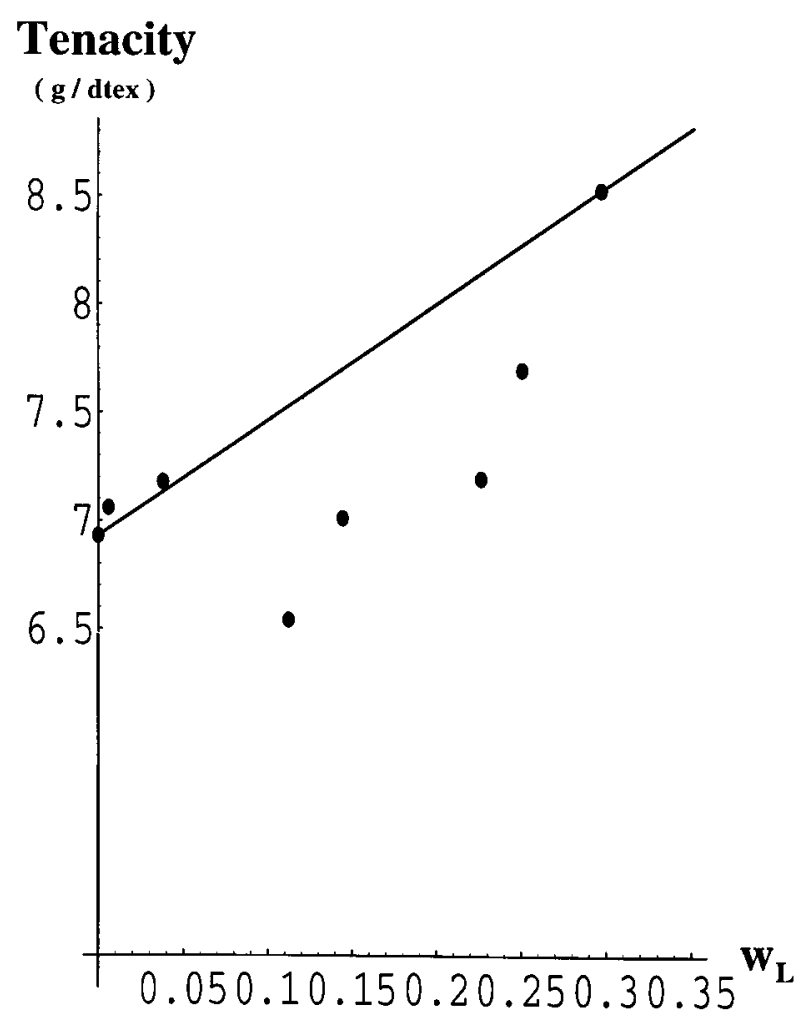

Figure 14 Plot of strength versus weight loss of $\mathrm{AD}$ using eq. (3).

Furthermore, a modified Rule of Mixtures can be adopted to deal with the cases in which interactions exist, ${ }^{12}$ as follows:

$$
X_{s}=X_{1} V_{1}+X_{2} V_{2}+\Delta X
$$

where $\Delta X$ is used to account for the effect on system property caused by the interactions. If $\Delta X$ $>0$, the interactions result in a positive or a synergetic effect, and $\Delta X<0$, a negative or reduced effect.

Turning to the data in this article, it will be noted that it was accumulated in terms of weight loss and not volume fractions of the system after hydrolysis. This renders a problem in analysis in that it is difficult to convert the weight loss into the volume fraction required in eq. (2) since it is extremely tedious to precisely determine the relative proportions and the distributions of the two components in the system at each level of hydrolysis. The irregular pattern of the dissolution process, as discussed before, further complicates the issue also.

As an alternative, we propose another empirical model to investigate the relationship between the system and component properties as a function of the weight loss, as follows:

$$
X_{s}=X_{s o}+a\left(W_{L}-W_{L o}\right) X_{1}
$$

where $X_{s o}$ is the original system property before the hydrolysis treatment, and $a$ is an interaction coefficient. $X_{s o}$ can be calculated from eq. (2) if all the required information is given, or is known experimentally, as shown in Tables II, IV, and V. $W_{L}$ is the weight loss and $W_{L o}$ is the starting point when data was collected. For instance, for the birefringence data in Table IV, $W_{L o}$ equals $11.2 \%$, and for the cases taken from Table $\mathrm{V}, W_{L o}$ equals $0 \%$. Also, $X_{1}$ is the original property of the core component (PET) when it is alone (CD). The interaction coefficient $a$ to be determined experimentally reflects the difference between various system properties. The larger the value of $a$, the greater the effect of the hydrolysis.

As the hydrolysis proceeds and the weight loss $W_{L}$ increases, the weaker, more flexible, and more extensible component (PBT) is first to dissolve away, and the contribution of the reinforcing component (PET) becomes increasingly significant, leading to an increase of system property repre-

\section{Extension at break}

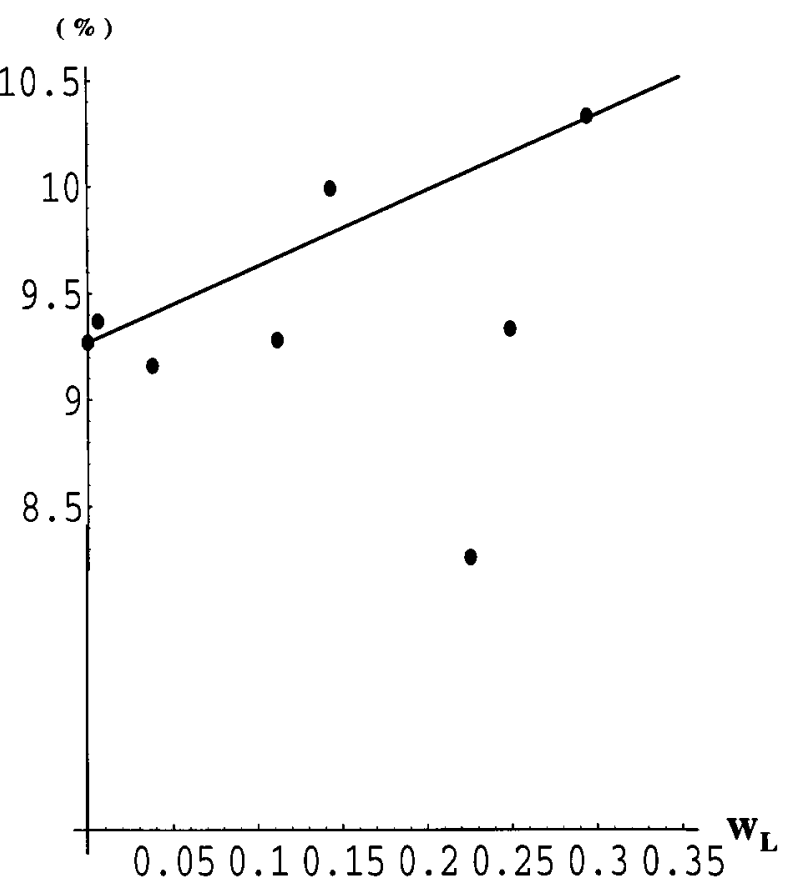

Figure 15 Plot of extension at break versus weight loss of $\mathrm{AD}$ using eq. (3). 


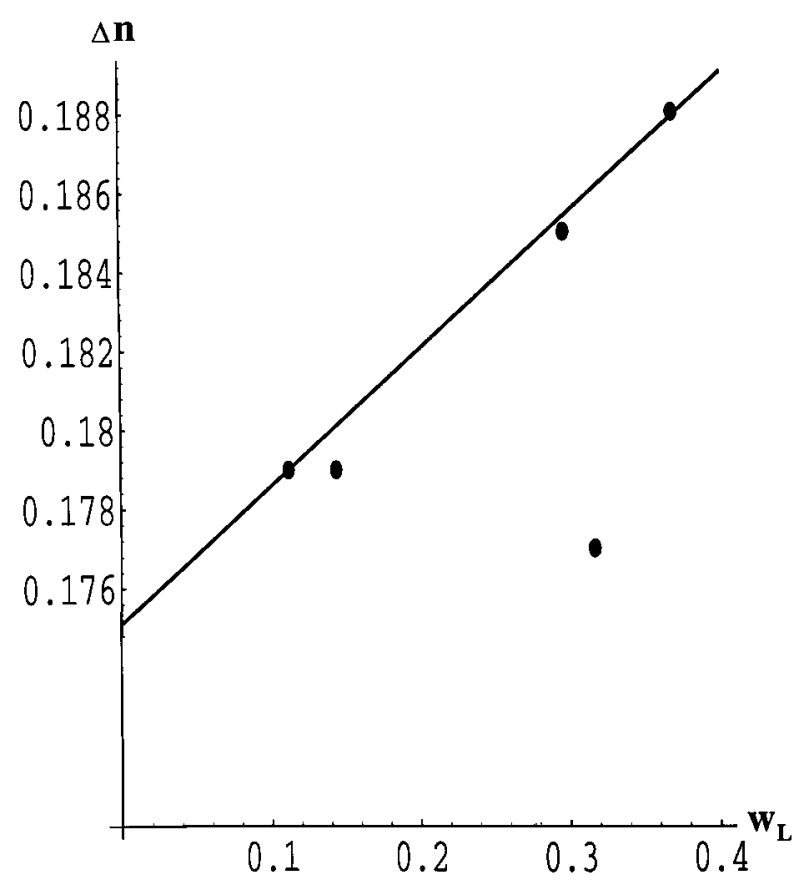

Figure 16 Plot of birefringence versus weight loss of $\mathrm{AD}$ using eq. (3).

sented by the term $\left(W_{L}-W_{L o}\right) X_{1}$. Equation (3) may thus provide an explanation for the synergy in system properties. It is more applicable when the reaction occurs primarily with the PBT, that is, roughly the first $40 \%$ of the hydrolysis.

Figures 14-16 are drawn with regression lines according to eq. (3) for tenacity, extension at break, and birefringence using the data in Tables IV and V. The values obtained for coefficient $a$ of the tenacity, extension at break, and birefringence are $0.8,0.23$, and 0.19 , respectively. Thus, this coefficient can be used to predict that the impact of the hydrolysis on properties is tenacity $>$ extension at break $\approx$ birefringence.

\section{CONCLUSIONS}

Alkaline hydrolysis can be used to study the fine structure of PBT-PET sheath-core bicomponent fibers, although the hydrolysis does not progress topochemically, as in the case of homofil PET and
PBT fibers. The birefringence, tenacity, breaking extension, and modulus of the bicomponent fiber increase after removal of its sheath. It appears that when the PBT sheath of a drawn PBT-PET bicomponent fiber is removed, the remaining PET fiber has enhanced tenacity when compared to a homofil PET spun and drawn in the same manner. An equation is proposed that includes an interaction parameter, which can be utilized to quantize which property is affected the most by the hydrolysis of a bicomponent system. In this instance, the order is strength $>$ extension at break $\approx$ birefringence. The alkaline hydrolysis appears to have revealed a structural nonuniformity that can be traced back to fiber formation. Thus, it has potential as a tool in assisting the development of improved bicomponent fibers.

The authors thank Hoechst-Celanese Corporation for financial support for this study.

\section{REFERENCES}

1. Collins, M. J.; Zeronian, S. H.; Semmelmeyer, M. J Appl Polym Sci 1991, 42, 2149.

2. Ellison, M. S.; Fisher, L. D.; Alger, K. W.; Zeronian, S. H. J Appl Polym Sci 1982, 27, 247.

3. Holmes, S. A.; Zeronian, S. H. J Macromol Sci, Pure Appl Chem 1994, A31, 1147.

4. Zeronian, S. H.; Buschle-Diller, G.; Inglesby, M. K. Polymer 1994, 35, 2587.

5. Ford, J. E.; Simmens, S. C. J Text Inst 1959, 50, 148.

6. ASTM D1505-85, Annu Book ASTM Stand 1989, Sect. 8, Vol. 08.01.

7. Ward, I. M.; Wilding, M. A.; Brody, H. J Polym Sci, Polym Phys Ed 1976, 14, 263.

8. Herlinger, H.; Gutmann, R.; Spindler, J. Chemiefasern-Textilindustrie 1989, 39/91, 947.

9. Tao, J.; Jin, H.-F.; Sun, T. Plast Rubber Compos Process Appl 1991, 16, 49.

10. Zeronian, S. H.; Collins, M. J.; Fisher, L. D.; Hawk, S. L. J Ind Fabrics 1985, 3(2), 19.

11. Nielsen, L. E. Predicting the Properties of Mixtures, Marcel Dekker: New York, 1978; p 8.

12. Pan, N.; Chen, K. Z.; Monego, C. J.; Backer, S. Tex Res J, submitted. 